\title{
Death, end-stage renal disease and renal function decline in patients with diabetic nephropathy in French cohorts of type 1 and type 2 diabetes
}

\author{
Samy Hadjadj ${ }^{1,2,3,4,17} \cdot$ Bertrand Cariou $^{5,6} \cdot$ Frederic Fumeron ${ }^{7,8,9} \cdot$ Elise Gand $^{10}$ • \\ Guillaume Charpentier ${ }^{11,12}$ - Ronan Roussel ${ }^{7,8,9}$ - Ahmed-Amine Kasmi ${ }^{1,3,4}$. \\ Jean-François Gautier ${ }^{7,8,13}$ - Kammel Mohammedi ${ }^{7,8,9}$ - Pierre Gourdy ${ }^{14,15,16}$. \\ Pierre-Jean Saulnier ${ }^{1,2,3,4}$. Eva Feigerlova ${ }^{1,2,3,4}$. Michel Marre ${ }^{7,8,9}$ on behalf of the \\ French JDRF Diabetic Nephropathy Collaborative Research Initiative (search for genes \\ determining time to onset of ESRD in T1D patients with proteinuria) and the \\ SURDIAGENE and DIABHYCAR study groups
}

Received: 29 April 2015 /Accepted: 15 September 2015 /Published online: 20 October 2015

(C) Springer-Verlag Berlin Heidelberg 2015

\begin{abstract}
Aims/hypothesis Microvascular complications are a common feature of diabetes but additional research is needed regarding diabetic nephropathy endpoints in type 1 and type 2 diabetes. Methods We compared 277 type 1 diabetes patients with 942 type 2 diabetes patients, with clinical proteinuria and no endstage renal disease (ESRD) at baseline, prospectively
\end{abstract}

The complete list of participating centres is available as electronic supplementary material (ESM).

Electronic supplementary material The online version of this article (doi:10.1007/s00125-015-3785-3) contains peer-reviewed but unedited supplementary material, which is available to authorised users.

Samy Hadjadj

samy.hadjadj@gmail.com

1 Université de Poitiers, UFR Médecine Pharmacie, Centre d'Investigation clinique, Poitiers, France

2 CHU de Poitiers, Service d'Endocrinologie-Diabetologie, Rue de la milétrie, 86000 Poitiers, France

3 CHU de Poitiers, Centre d'Investigation Clinique, Poitiers, France

4 Inserm, CIC1402 and U1082, Poitiers, France

5 Department of Endocrinology, University Hospital of Nantes, Nantes, France

6 Inserm, UMR1087-CNRS UMR6291, Institut du Thorax, Nantes, France

7 Université Paris Diderot, Sorbonne Paris Cité, Paris, France followed for death, ESRD and decline in estimated glomerular filtration rate (eGFR, all available measures).

Results The incidence rate of death was 67.0 (95\% CI 59.2, $74.8)$ vs $24.6(95 \%$ CI, $19.0,30.2)$ per 1,000 patient-years, in type 2 diabetes and type 1 diabetes, respectively. Unadjusted risk for death was greater for type 2 diabetes patients (HR 3.423; 95\% CI, 2.501, 4.683; $p<0.0001$ ), but the difference
8 Inserm, UMRS1138, Centre de Recherche des Cordeliers, Paris, France

9 Diabetologie, Endocrinologie Nutrition, DHU FIRE, Hopital Bichat, AP-HP, Paris, France

10 CHU de Poitiers, Pôle Dune, Poitiers, France

11 Centre Hospitalier Sud Francilien, Corbeil-Essonnes, France

12 CERITD, Evry, France

13 Department of Diabetes and Endocrinology, Assistance Publique Hôpitaux de Paris, Lariboisière Hospital, Paris, France

14 CHU de Toulouse, Service de Diabétologie, Maladies Métaboliques et Nutrition, Toulouse, France

15 Inserm, UMR1048/I2MC, Toulouse, France

16 Université Paul Sabatier, UMR1048/I2MC, Toulouse, France

17 CHU de Poitiers, Service d'Endocrinologie-Diabetologie and Centre d'Investigation Clinique, Rue de la Milétrie, 86000 Poitiers, France 
did not persist after adjustment for age $\left(\mathrm{HR}_{\text {age-adj }} 0.859 ; 95 \%\right.$ CI $0.581,1.269 ; p=0.445)$. The incidence rate of ESRD was $18.4(95 \%$ CI $14.2,22.5)$ vs 47.1 (95\% CI 38.4, 55.9) per 1,000 patient-years, in type 2 diabetes and type 1 diabetes, respectively. Unadjusted risk for ESRD was lower in type 2 diabetes (HR 0.399; 95\% CI 0.287, 0.554; $p<0.0001$ ), but the difference did not persist after adjustment for sex, age and baseline serum creatinine $\left(\mathrm{HR}_{\mathrm{adj}} 0.989 ; 95 \%\right.$ CI 0.597, 1.639; $p=0.965$ ). In a mixed linear model, eGFR decline was not significantly different in type 2 vs type 1 diabetes (difference in slope $-0.19[0.28] \mathrm{ml} \mathrm{min}^{-1} 1.73 \mathrm{~m}^{-2}$ year $^{-1} ; p=0.512$ ). Conclusions/interpretation In diabetic nephropathy, once baseline risk factors were taken into account the risk for death, ESRD and renal function decline did not significantly differ between type 1 diabetes and type 2 diabetes.

Keywords Death · Diabetic complications · Diabetic nephropathy $\cdot$ Renal function decline $\cdot$ Type 1 diabetes $\cdot$ Type 2 diabetes

\begin{tabular}{|c|c|}
\hline \multicolumn{2}{|l|}{ Abbreviations } \\
\hline CKD & Chronic kidney disease \\
\hline CKD-EPI & $\begin{array}{l}\text { Chronic Kidney Disease Epidemiology } \\
\text { Collaboration }\end{array}$ \\
\hline DIABHYCAR & $\begin{array}{l}\text { Non-insulin-dependent diabetes hyperten- } \\
\text { sion, microalbuminuria or proteinuria, } \\
\text { cardiovascular events, and ramipril }\end{array}$ \\
\hline eGFR & Estimated glomerular filtration rate \\
\hline ESRD & End-stage renal disease \\
\hline GENEDIAB & Genes nephropathy and diabetes \\
\hline GENESIS & Genes nephropathy and sib-pair study \\
\hline IQR & Interquartile range \\
\hline SURDIAGENE & Survival diabetes and genetics \\
\hline $\mathrm{UAC}$ & Urinary albumin concentration \\
\hline
\end{tabular}

\section{Introduction}

Diabetes is a chronic condition, the incidence of which is rapidly rising throughout the world. While this increase is particularly well known for type 2 diabetes, it has also been noted for type 1 diabetes. In both forms of the disease, the common feature is chronic hyperglycaemia, leading to microvascular complications [1]. The presence of microvascular disease is the key element defining the blood glucose threshold for diabetes [1]. Renal complications represent a major turning point in the lives of people with diabetes. In type 1 diabetes, the presence of diabetic nephropathy is associated with increased mortality risk, while non-nephropathic individuals have a more favourable life expectancy [2, 3]. Similarly, in type 2 diabetes, nephropathy is associated with increased cardiovascular mortality and morbidity $[4,5]$. Moreover, diabetic kidney disease is considered to be the leading cause of end-stage renal disease (ESRD) in westernised countries, including the USA (www.usrds.org, accessed 22 September 2015).

Although the classification of type 1 and 2 diabetes can be difficult, and is sometimes the subject of debate [6], the consequences of the type of diabetes on renal complications are poorly established. In the pathophysiological relationship between diabetes and its complications, type 1 diabetes mainly involves glucose injury, while renal disease in type 2 diabetes is generally considered more heterogeneous, including factors other than glucose such as lipids, inflammation or blood pressure. The pattern of renal decline according to the type of diabetes has not been adequately studied and refers primarily to a single highly referenced paper, published more than 25 years ago [7].

The systematic use of blockers of the renin-angiotensinaldosterone system, the launching of short-acting and longacting insulin analogues promoting enhanced glucose control and new techniques for statistical analysis have led us to reconsider occurrences of end-stage complications in type 1 and type 2 diabetes. In addition, decline in renal function is emerging as an important outcome [8,9]. Given the existence of modern therapies, analysis of contemporaneous data on the rates of renal function decline in diabetes could help to design trials and anticipate needs for renal replacement strategy. In the context of the international effort to evaluate the genetic factors involved in diabetic nephropathy, it is important to determine whether data generated from type 2 diabetes patients can be used for type 1 diabetes and vice versa.

We have consequently examined the relationship between, on the one hand, type of diabetes and ESRD/mortality risk and, on the other hand, renal function decline, using several cohorts, totalling more than 1,200 patients with diabetic nephropathy, defined clinically by established proteinuria.

\section{Methods}

\section{Patients}

Individuals of Europid ethnicity with type 1 diabetes were recruited on the occasion of the GENEDIAB (genes nephropathy and diabetes) and GENESIS (genes nephropathy and sibpair study) studies $[10,11]$. All type 1 diabetes patients attending diabetes clinics were invited to be included in the GENEDIAB study, provided they had severe diabetic retinopathy (proliferative or severe non-proliferative requiring panphotocoagulation), regardless of their nephropathy status. All type 1 diabetes patients with retinopathy and diabetes duration longer than 15 years were invited to be included in the GENESIS study. All consecutive non-duplicate type 1 diabetes patients in Corbeil-Essonnes, Nantes, Paris Saint-Louis, 
Poitiers and Toulouse were recruited for an observational study.

A secondary selection was applied among included patients: all patients diagnosed with proteinuria with or without renal failure were followed for risk of renal function decline, ESRD and mortality, on the occasion of the JDRF Diabetic Nephropathy Collaborative Research Initiative (see Acknowledgements). All of these cohorts were hospital based.

Individuals of Europid ethnicity with type 2 diabetes and who had proteinuria were selected from two independent cohorts-SURDIAGENE (survival diabetes and genetics) and DIABHYCAR (non-insulin-dependent diabetes, hypertension, microalbuminuria or proteinuria, cardiovascular events, and ramipril) - for which an adjudication committee reviewed study outcomes.

SURDIAGENE participants were consecutively recruited at Poitiers University Hospital as part of an inception cohort, performed at the Poitiers University Hospital. Organisation of follow-up regarding renal outcomes has been described previously [12]. Briefly, all type 2 diabetes patients attending the Poitiers diabetes clinic were invited to take part in a follow-up observational study. Non-inclusion criteria were evidence of non-diabetic renal disease (according to the investigator's opinion) and residing outside the Poitou Charentes region.

The design and results of the DIABHYCAR study have been reported previously $[13,14]$. The participants of the current analysis were people with type 2 diabetes on oral medication, aged $\geq 50$ years, with serum creatinine $\leq 150 \mu \mathrm{mol} / 1$ and two consecutive urine samples with albumin concentration $\geq 20 \mathrm{mg} / \mathrm{l}$, followed by their general practitioner. This was a double-blind, parallel, 4 year design, evaluating a low dose of ramipril ( $1.25 \mathrm{mg} /$ day) compared with placebo, with a primary endpoint combining the occurrence of cardiovascular and renal events (end-stage renal failure).

All participants in this research signed an informed consent form. Ethics committees approved the designs of all the different studies. Clinical variables collected at baseline included age, age at diabetes onset, blood pressure and smoking status.

\section{Definition of diabetes}

Diabetes classification was made using American Diabetes Association diagnostic criteria, as defined in 1997. Type 1 diabetes was defined as age at diabetes onset before 35 years (GENESIS/GENEDIAB) or 40 years (all five other cohorts) and a definitive requirement for insulin treatment less than 1 year following diagnosis. Type 2 diabetes was defined by age at diabetes onset older than 40 years, absence of ketoacidosis and glycaemic control without insulin treatment for at least 2 years after diagnosis. Patients with clinical suspicion of secondary diabetes were excluded.

\section{Definition of diabetic nephropathy}

We focused on type 1 diabetes patients with proteinuria, defined as urinary albumin excretion rate higher than 300 $\mathrm{mg} / 24 \mathrm{~h}$ on two out of three consecutive sterile urine collections.

In the SURDIAGENE cohort, diabetic nephropathy was established on two out of three consecutive sterile urine collections considering urinary albumin-to-creatinine ratio higher than $30 \mathrm{mg} / \mathrm{mmol}$.

In the DIABHYCAR, selection criteria included increased urinary albumin concentration (UAC) on two consecutive occasions equal to or higher than $20 \mathrm{mg} / \mathrm{l}$. We included patients with diabetic nephropathy, diagnosed when UAC was above $200 \mathrm{mg} / \mathrm{l}$.

\section{Clinical endpoints}

Our primary fatal clinical endpoint was all-cause death. Hospital records were analysed for identification of the presence/ date of the endpoints; where details were missing, general practitioners were interviewed by telephone. When no data were present, the national death certificate registry was consulted.

Our co-primary non-fatal clinical endpoint was ESRD as defined by renal replacement therapy (kidney graft or dialysis or a sustained estimated glomerular filtration rate [eGFR] below $10 \mathrm{ml} \mathrm{min}^{-1} 1.73 \mathrm{~m}^{-2}$ ). We took into account the different time intervals between severe renal failure (below $10 \mathrm{ml} \mathrm{min}^{-1}$ $1.73 \mathrm{~m}^{-2}$ ) and time of dialysis onset, which might arise from non-medical considerations.

Our secondary outcome was the trajectory of eGFR. We considered a linear regression to compute annual eGFR slope. A slope of $\leq-5 \mathrm{ml} \mathrm{min}^{-1} 1.73 \mathrm{~m}^{-2}$ per year defined rapid renal function decline, according to KDIGO (kidney disease improving global outcomes) [15]. In type 1 diabetes, prospective data only were considered for chronic kidney disease (CKD) stage 1-2 patients while prospective and retrospective data were used to build eGFR trajectories in CKD stage 3-5 patients. Only prospective data were considered for type 2 diabetes study participants.

\section{Biological determinations}

Type 1 diabetes Patients from the GENEDIAB and GENESIS cohorts had their biological samples determined centrally at baseline for serum creatinine, $\mathrm{HbA}_{1 \mathrm{c}}$ and urinary albumin. Patients from other cohorts of type 1 diabetes patients had their blood and urine samples handled locally.

During follow-up, serum creatinine was determined locally using a colorimetric method. Different kits were used according to local practice. The patients' records were examined and every locally determined serum creatinine concentration was 
used to build eGFR trajectories. Only the serum creatinine determined prior to ESRD was taken into account.

Type 2 diabetes In the SURDIAGENE cohort, urinary creatinine was measured on a Hitachi 911 automatic analyzer (Roche Diagnostics, Meylan, France). Serum creatinine and urinary albumin were measured by nephelometry on a Modular System P (Roche Diagnostics). $\mathrm{HbA}_{1 \mathrm{c}}$ was determined by an HPLC method using an ADAMS A1C HA-8160 analyzer (Menarini, Florence, Italy) (normal values 4.0-6.0\% [20-42 $\mathrm{mmol} / \mathrm{mol}])$.

In the DIABHYCAR cohort, $\mathrm{HbA}_{1 \mathrm{c}}$ was determined centrally using an HPLC method (Biorad DIAMAT Analyser; Bio-Rad, Richmond, CA, USA) with normal values ranging from 4.0 to $5.6 \%(20-38 \mathrm{mmol} / \mathrm{mol})$. Locally determined serum creatinine concentrations were collected yearly, in accordance with study protocol. Glomerular filtration rate was estimated using the Chronic Kidney Disease Epidemiology Collaboration (CKD-EPI) 2009 creatinine equation [16].

\section{Statistical analysis}

Data were stored and analysed using SAS 9.3 (SAS Systems, Cary, NC, USA). Continuous variables were summarised by means and SDs or by median and interquartile range (IQR) according to data distribution. Categorical variables were presented by frequencies and percentages. Standard univariate statistical methods were used to compare characteristics in type 1 vs type 2 diabetes.

Time to clinical endpoints (all-cause death or ESRD) was plotted according to the type of diabetes with Kaplan-Meier curves, and comparison was made using the logrank test. Univariate and multivariate Cox proportional hazard models were used to identify the effect of key covariates. Covariates with nominal statistical significance were used in multivariate analysis. The result was expressed as the HR and its $95 \%$ CI. The Fine and Gray model was evaluated to analyse competing risks of death in the ESRD risk model. The result was expressed as the sub-HR and its $95 \% \mathrm{CI}$.

As no data were available to address the research question, no formal power calculation was performed prior to the analysis.

Analysis of renal function decline was performed using a mixed linear model, taking repeated longitudinal eGFR data into account so as to model the trajectories of eGFR over time according to type of diabetes, adjusted on several covariates. The random errors of the mixed linear analysis we conducted were defined as a random intercept and slope. The coefficients were presented with their standard error. The mixed model was applied only to individuals with at least two determinations during follow-up and before ESRD (see flow chart presented in Fig. 1). To determine whether type 1 diabetes evolves differently from type 2 diabetes over time, an interaction term between time and type of diabetes was added in the mixed model.

In all the multivariable models employed, variables with univariate statistical significance were applied, using a backward selection procedure. Statistical significance was set at $p<0.05$.

\section{Results}

\section{Baseline characteristics}

For type 2 diabetes, 1,468 consecutive participants, including 232 proteinuric patients, entered the SURDIAGENE inception cohort. In the DIABHYCAR population, we restricted our study to French participants. The population entering the selection was made up of 20,296 participants with type 2 diabetes. Among them, 3,137 were randomised and we focused our analysis on the 710 proteinuric patients. In the population of type 1 diabetes patients, the whole population suitable for a case-control approach (with disease duration $>15$ years) consisted of 1,367 participants, including the 456 nephropathic patients detailed in Fig. 1.

A total of 1,219 patients were available for analysis of the primary endpoint, consisting of 942 with type 2 diabetes and 277 with type 1 diabetes.

Baseline clinical and biological characteristics of the study population are summarised in Table 1 and electronic supplementary material (ESM) Tables 1 and 2. Comparatively, patients with type 2 diabetes had a higher BMI while those with type 1 diabetes were younger and had longer diabetes duration. Baseline eGFR was lower in patients with type 2 diabetes than in those with type 1 diabetes.

Follow-up duration was longer in type 1 diabetes compared with type 2 diabetes, in accordance with calendar year of recruitment (type 2 diabetes: DIABHYCAR, range 19951998 [median 1996]) and SURDIAGENE, range 2002-2011 [median 2004]; type 1 diabetes: range 1994-2012 [median 1995]), corresponding to a median (IQR) follow-up duration of 56 (26) months in type 2 diabetes and 143 (106) months in type 1 diabetes.

\section{All-cause death}

There were 358 all-cause deaths during follow-up: 285 and 73 translating to an incidence rate of $67.0(95 \%$ CI $59.2,74.8)$ vs 24.6 (95\% CI $19.0,30.2)$ per 1,000 patient-years, in type 2 diabetes and type 1 diabetes, respectively.

In Fig. 2 we plotted Kaplan-Meier curves for the risk of allcause death according to the type of diabetes. ESM Table 3 


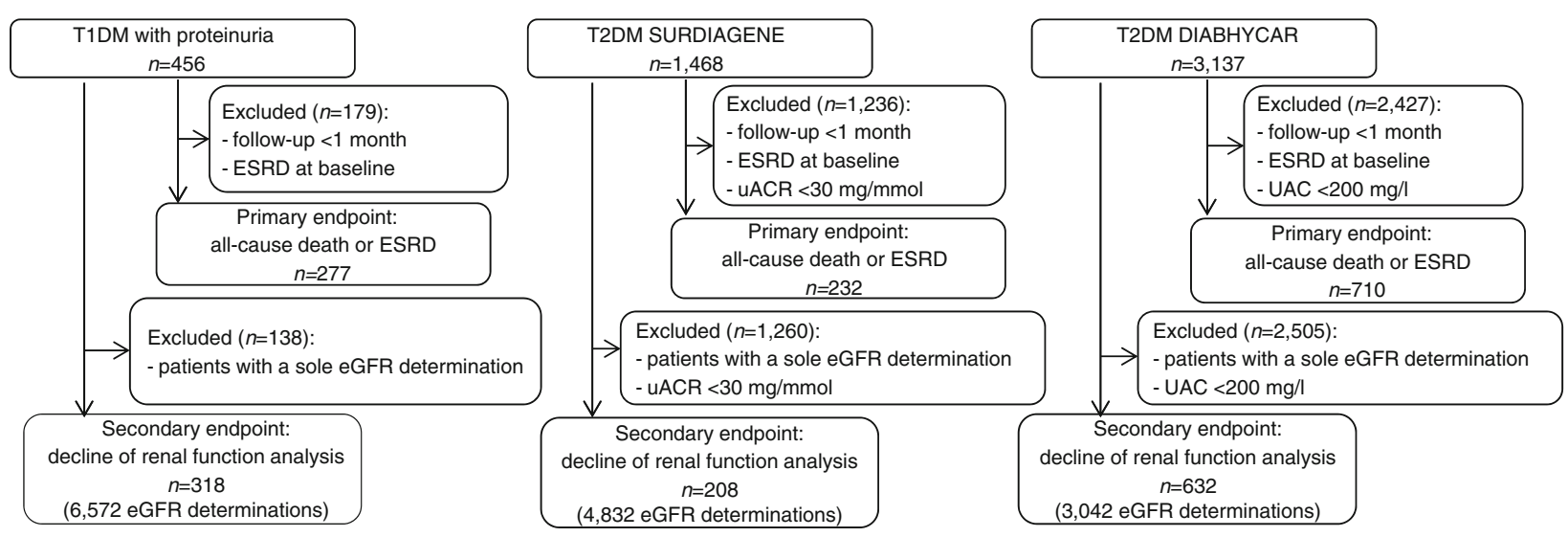

Fig. 1 Flow chart of study participants. T1DM, type 1 diabetes mellitus; T2DM, type 2 diabetes mellitus; uACR, urinary albumin-creatinine ratio

summarises the different covariates associated with risk for death.

In a Cox multivariate model, the risk for all-cause death was significantly greater for type 2 diabetes patients (HR

Table 1 Baseline clinical and biological characteristics of study participants

\begin{tabular}{|c|c|c|c|}
\hline Variable & $\begin{array}{l}\text { Type } 1 \\
\text { diabetes }\end{array}$ & $\begin{array}{l}\text { Type } 2 \\
\text { diabetes }\end{array}$ & $p$ value \\
\hline & $n=277$ & $n=942$ & \\
\hline Sex, male, $n(\%)$ & $163(59)$ & $666(71)$ & 0.0002 \\
\hline Age, years & $42.1 \pm 11.5$ & $66.3 \pm 8.7$ & $<0.0001$ \\
\hline Follow-up duration, months & $143(106)$ & $56(26)$ & $<0.0001$ \\
\hline BMI, $\mathrm{kg} / \mathrm{m}^{2}$ & $24.1 \pm 3.4$ & $30.0 \pm 5.3$ & $<0.0001$ \\
\hline Active smoking, $n(\%)$ & $83(30)^{\mathrm{a}}$ & $158(19)^{\mathrm{b}}$ & $<0.0001$ \\
\hline UAE, $\mathrm{mg} / 24 \mathrm{~h}$ & $516(1,068)$ & - & \\
\hline $\mathrm{ACR}, \mathrm{mg} / \mathrm{mmol}$ & & $112.3(166.3)$ & \\
\hline Diabetes duration, years & $26.3 \pm 9.9$ & $16.2 \pm 8.7$ & $<0.0001$ \\
\hline $\mathrm{HbA}_{1 \mathrm{c}}, \%$ & $8.9 \pm 1.8$ & $8.0 \pm 1.7$ & $<0.0001$ \\
\hline $\mathrm{HbA}_{1 \mathrm{c}}, \mathrm{mmol} / \mathrm{mol}$ & $73.8 \pm 20.9$ & $63.9 \pm 19.7$ & $<0.0001$ \\
\hline 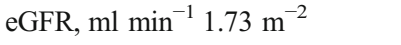 & $71.9 \pm 27.3$ & $67.6 \pm 22.4$ & 0.0174 \\
\hline CKD stage, $n(\%)$ & & & $<0.0001$ \\
\hline 1 & $57(21)$ & $168(18)$ & \\
\hline 2 & $77(28)$ & $422(45)$ & \\
\hline 3 & $109(38)$ & $295(31)$ & \\
\hline 4 & $27(10)$ & $44(5)$ & \\
\hline 5 & $7(3)$ & $13(1)$ & \\
\hline Systolic blood pressure (mmHg) & $144.0 \pm 19.2$ & $146.5 \pm 15.8$ & 0.0578 \\
\hline Diastolic blood pressure (mmHg) & $81.6 \pm 11.1$ & $80.9 \pm 10.2$ & 0.3747 \\
\hline Any antihypertensive drug, $n(\%)$ & $230(83)$ & $691(73)$ & $<0.0001$ \\
\hline ACE-I use, $n(\%)$ & $201(73)$ & $169(18)$ & $<0.0001$ \\
\hline ARB use, $n(\%)$ & $17(7)$ & $71(8)$ & $<0.0001$ \\
\hline
\end{tabular}

Quantitative variables are shown as mean $\pm \mathrm{SD}$ or median (IQR)

${ }^{\text {a }}$ Data missing in 29 patients

${ }^{\mathrm{b}}$ Data missing in 87 patients

ACE-I, ACE inhibitors; ACR, albumin-creatinine ratio; ARB, angiotensin receptor blockers
3.423; 95\% CI 2.501, 4.683; $p<0.0001)$ but this difference did not persist after adjustment for age $\left(\mathrm{HR}_{\text {age-adj }} 0.859\right.$; 95\% CI 0.581, 1.269; $p=0.445)$.

\section{Renal outcomes}

ESRD During follow-up, ESRD occurred in 75 patients with type 2 diabetes and 112 with type 1 diabetes, translating to an incidence rate of 18.4 (95\% CI 14.2, 22.5) vs 47.1 (95\% CI $38.4,55.9)$ per 1,000 patient-years in type 2 diabetes vs type 1 diabetes, respectively. Figure 3 describes the relationship between the type of diabetes and ESRD risk with a plotted Kaplan-Meier curve.

Data for the univariate Cox proportional hazard model are summarised in ESM Table 4. The crude risk for ESRD was significantly lower in type 2 diabetes (HR 0.399; 95\% CI $0.287,0.554 ; p<0.0001)$, but the difference did not persist after adjustment for sex, age and baseline serum creatinine $\left(\mathrm{HR}_{\mathrm{adj}} 0.989 ; 95 \%\right.$ CI $\left.0.597,1.639 ; p=0.965\right)$.

When using a competing risk model, with ESRD as primary outcome and death as competing risk, we found roughly unchanged results with no effect of type of diabetes on risk of ESRD (ESM Table 5).

Renal function decline We collected 4,832 serum creatinine determinations for 208 individuals with type 2 diabetes (median 27 per patient) from the SURDIAGENE

Table 2 Multivariate mixed linear model including random intercept and slope

\begin{tabular}{llll}
\hline Variable & Coefficient & SD & $p$ value \\
\hline Intercept & 83.76 & 1.95 & $<0.0001$ \\
Time (years) & -2.6 & 0.14 & $<0.0001$ \\
T2DM patients (reference T1DM) & 28.74 & 1.98 & $<0.0001$ \\
Age (years) & -0.72 & 0.03 & $<0.0001$ \\
\hline
\end{tabular}

T1DM, type 1 diabetes mellitus; T2DM, type 2 diabetes mellitus 


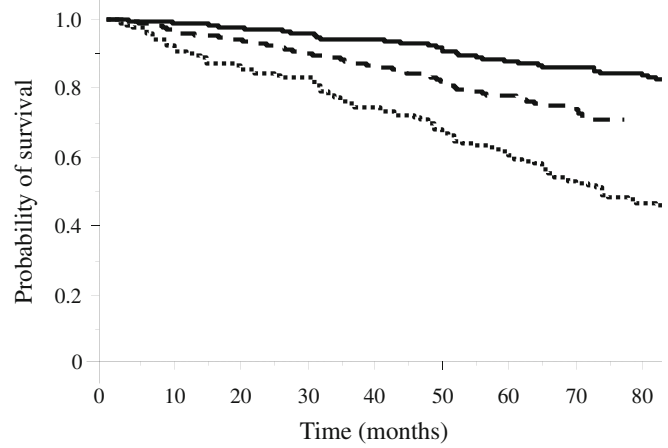

Number at risk

T1DM

T2DM DIABHYCAR

277
710
232

263
654

245
568

221

Fig. 2 Kaplan-Meier curves showing survival from all-cause death in the original three cohorts. Solid line, type 1 diabetes patients; dashed line, type 2 diabetes from the DIABHYCAR cohort; dotted line, type 2 diabetes from the SURDIAGENE cohort. Logrank 117, $p<0.0001$. T1DM, type 1 diabetes mellitus; T2DM, type 2 diabetes mellitus

cohort, 3,042 for 632 individuals with type 2 diabetes (median 5 per patient) from the DIABHYCAR cohort and 6,572 for 318 patients with type 1 diabetes (median 17 per patient). When considering the prospective approach in type 1 diabetes alone, 36 patients were excluded from the analysis.

Table 2 summarises the mixed linear model with random intercept and slope and indicates that patients with type 2 diabetes have an eGFR that is $28.7 \mathrm{ml} \mathrm{min}{ }^{-1} 1.73 \mathrm{~m}^{-2}$ higher at baseline than that of type 1 diabetes patients, adjusted on age. Annual decline was not significantly different according to type of diabetes: difference in slope $-0.19 \pm 0.28 \mathrm{ml} \mathrm{min}{ }^{-1}$ $1.73 \mathrm{~m}^{-2}$ per year; $p=0.512$. When including baseline eGFR as a random variable in the mixed linear model, we still found no difference in trajectory according to type of diabetes.

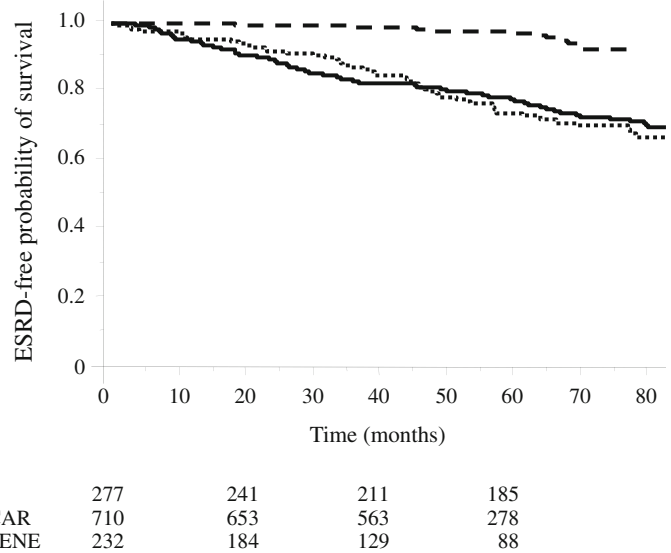

Number at risk

T1DM

T2DM DIABHYCAR

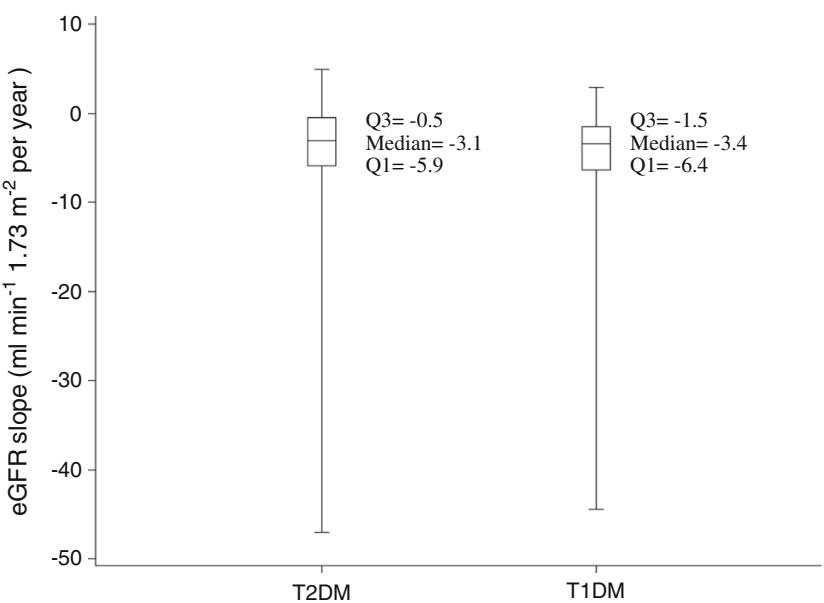

Fig. 4 Representation of annual eGFR slopes, according to the type of diabetes. Boxes indicate 25 th and 75 th percentiles, centred on the median, and whiskers represent minimum and maximum values

The plot of eGFR slopes according to type of diabetes is shown in Fig. 4. When we compared patients according to rapid decline in renal function, we found no difference according to type of diabetes: $251 / 840$ in type 2 vs $107 / 318$ in type 1 diabetes patients $\left(\chi^{2}=1.53 ; p=0.216\right)$.

\section{Discussion}

This study was designed to evaluate risk for all-cause death and renal function in more than 1,200 people with diabetic nephropathy. We particularly wished to determine whether the risk for ESRD and the decline in renal function differed according to the type of diabetes. Our data showed that the crude rate of death was greater in type 2 diabetes patients while the crude rate of ESRD was greater in type 1 diabetes patients. Age-adjusted risk for death did not differ according to type of diabetes, while the increased risk for ESRD in type 1 diabetes did not persist after adjustment for baseline eGFR. Accordingly, using a multiple linear model, we found no significant difference in renal decline according to the type of diabetes.

Our data are in concordance with those of the UKPDS (UK prospective diabetes study), for whom risk for death was significantly higher than risk for ESRD in patients with type 2 diabetes and proteinuria [17] and with data from the FinnDiane study, which found (in 592 proteinuric type 1 diabetes patients, followed for 10 years) a higher number of patients having reached ESRD $(n=210)$ than those having died $(n=56)$ [18].

The main findings in this study are that risk for death and annual rate of renal function decline were not significantly different according to the type of diabetes, provided that key variables such as age, sex and baseline serum creatinine are taken into account, considering two complementary statistical

Fig. 3 Kaplan-Meier curves showing survival without ESRD in the three original cohorts. Solid line, type 1 diabetes patients; dashed line, type 2 diabetes from the DIABHYCAR cohort; dotted line, type 2 diabetes from the SURDIAGENE cohort. Logrank 102, $p<0.0001$. T1DM, type 1 diabetes mellitus; T2DM, type 2 diabetes mellitus 
approaches: mixed linear model and competing risk analysis. To the best of our knowledge, our study is the first prospective large-scale study to comprehensively examine this question, even though some previous small retrospective studies have suggested the similarity in renal outcome risk according to the type of diabetes $[19,20]$.

The data presented here are consistent with the data from Hasslacher et al suggesting a similar risk for the development of proteinuria in type 2 and type 1 diabetes, even though these data were obtained more than 25 years ago, with different standards of care [7].

The eGFR intercept differed between patients with type 1 and those with type 2 diabetes, suggesting that the former had a more serious decline in eGFR at baseline. Interestingly, this was not driven by the DIABHYCAR cohort, in which patients were selected on the basis of mildly decreased renal function (serum creatinine below $150 \mu \mathrm{mol} / \mathrm{l}$ ). Comparison between type 1 and type 2 diabetes patients from the SURDIAGENE study produced a similar result but with a lower eGFR intercept (data not shown), suggesting that the same rate of renal function decline according to type of diabetes was not influenced by selection criteria in the two cohorts of type 2 diabetes patients. Whether or not proteinuria appears in type 1 diabetes patients at a lower eGFR is beyond the scope of this paper but deserves further investigation.

Some limitations to our study must be acknowledged. We were not able to study, as had others [7], all the patients recruited in the same centre. On the other hand, as the whole population was recruited from the same country, we achieved a good consistency in applied clinical guidelines. A second limitation is that while all the patients were all selected with proteinuria at baseline, time to proteinuria onset was not known. Since the object of consideration in this study was time to clinical endpoints, possible modification of our findings on account of past renal disease is hard to establish. However, the analysis of renal function using mixed linear model helps to take renal function decline into consideration and to circumvent the question of time of onset of proteinuria. We defined type of diabetes on a clinical basis, as previously validated [21]. The finding that patients with type 2 vs type 1 diabetes were older and more obese can be seen as reassuring, but classification of diabetes per se has been criticised [6]. Finally, issues regarding biological determinations in type 1 diabetes patients must be acknowledged, as serum creatinine was determined locally using different methods. Although different kits were used, all of the determinations were based on picric acid methods in patients with type 1 diabetes. Furthermore, eGFR computed with the MDRD (modification of diet in renal disease) study equation instead of the CKD-EPI formula produced similar results (data not shown).

The strengths of our study were largely due to the largesized population of proteinuric patients and a prospective longitudinal design. At variance with retrospective data, which are subject to survival bias, we could consequently perform competing risk analysis of death in addition to ESRD. Finally, the linear model, which had not been previously available, was particularly suited to examination of the present question.

Whether our findings can be generalised is an important question. In type 1 diabetes, the proportion of patients with CKD stage 4 or 5 was $2.5 \%$, quite similar to the $2.3 \%$ found in a population-based evaluation of French type 1 diabetes patients [22]. Regarding the representativeness of the studied type 2 diabetes patients, general practitioners recruited those participating in the DIABHYCAR study. This makes the generalisability good, since about $90 \%$ of the type 2 diabetes patients are cared for by general practitioners in mainland France (www.invs.sante.fr/Dossiers-thematiques/maladieschroniques-et-traumatismes/Diabete/Etudes-Entred/EtudeEntred-2007-2010/Resultats-epidemiologiques-principaux-dEntred-metropole, Accessed 22 September 2015). To enrich this cohort with data from patients with proteinuria, we added on those under the care of our tertiary referral centre participating in the SURDIAGENE study [23], for whom the standard of care is more contemporary than for those patients included in the DIABHYCAR study. Interestingly, the results from the hospital-based cohort (SURDIAGENE) were close to those from the general practitioner-based clinical trial (DIABHYCAR), while the prevalence of proteinuric cases differed largely between hospital-based patients (approximately 15\%) and general practitioner-recruited patients (approximately $3.5 \%$ ). We consequently believe that our results are likely to be generalisable in patients with proteinuria, even though the replication of our results using registry- or population-based cohorts would be of great interest.

One key question arises from our study: do our findings establish whether any risk factor or biomarker used for type 1 diabetes can also be used for type 2 diabetes patients, and vice versa? This remains an open question particularly for genetic risk factors. Of note, recent genome-wide association studies have identified some new genetic risk factors in type 1 diabetes that were subsequently established in patients with type 2 diabetes $[24,25]$. Furthermore, a recent report suggested a strong concordance for gene transcripts in glomeruli (94\%) between type 1 and type 2 diabetes in murine experimental models, while no high concordance (55\%) was found for gene transcripts in sciatic nerve [26], suggesting concordant mechanisms for renal complications in both types of diabetes but not for all microvascular complications.

To conclude, our results support the hypothesis, although clear differences exist between type 1 and type 2 diabetes in terms of complications. Even though there is a huge difference in unadjusted risk for ESRD and for all-cause death, renal function decline is not significantly different in type 1 and type 2 diabetes patients with diabetic nephropathy. 
Acknowledgements All participants in the cohorts are warmly thanked for their contribution. We also wish to thank J. Arsham (CHU Poitiers) for rereading and editing our original English language manuscript.

Some of the data were presented as an abstract at the 75th Scientific Sessions of the American Diabetes Association in 2015.

Statistical analysis was carried out by D. A. Tregouet, M. Germain, M.-L. Franchignard and B. Gyorgy (UMR S 937, Pitié Salpétrière, Paris) and E. Gand (Pôle Dune, CHU Poitiers).

Biological sample management was performed at the following centres: GENEDIAB and GENESIS - Inserm U1138, Paris (M. Marre; N. Bellili); Corbeil-Essonnes Diabetes Center, UMR 8090, Lille (P. Froguel, S. Gagné); Nantes - Paris St Louis - Toulouse Diabetes Center - ISIS Study Kremlin Bicêtre, Inserm U 986 (P. Bougnères, D. Fradin, S. Le Fur); Poitiers Diabetes Center, Inserm CIC1402 and CRB 0033-00068, Poitiers (S. Hadjadj, P. J. Saulnier, S. Brishoual). A. Pavy, J. Guignet and M.-C. Pasquier (CHU Poitiers) helped with the collection of serum creatinine data in the SURDIAGENE cohort.

Data in type 1 diabetes patients are presented on behalf of the study group entitled 'genes responsible for rapid progression to ESRD in type 1 diabetes' (A. Krolewski, Joslin Diabetes Center, Boston, MA, USA), work package of the JDRF Diabetic Nephropathy Collaborative Research Initiative.

Principal investigators of the cohorts were as follows: GENESIS, GENEDIAB and DIABHYCAR: M. Marre (Bichat, Paris), P. Gourdy (Toulouse Diabetes Center), G. Charpentier (Corbeil-Essonnes Diabetes Center), J. F. Gautier (Paris Saint-Louis Diabetes Center, Paris Lariboisière), B. Cariou (Nantes Diabetes Center); SURDIAGENE: S. Hadjadj (Poitiers Diabetes Center).

The complete list of participating centres is available in the ESM.

Funding This work in type 1 diabetes patients was made possible thanks to the support of the JDRF grant 17-2013-8.

The SURDIAGENE cohort was supported by grants from the French Ministery of Health (PHRC-Poitiers 2004; PHRC-IR 2008), Association Française des Diabétiques (AFD; Research Grant 2003) and Groupement pour l'Etude des Maladies Métaboliques et Systémiques (GEMMS Poitiers, France)

The original DIABHYCAR trial was supported by grants from Sanofi-Aventis (France), the French Ministry of Health (Programme Hospitalier de Recherche Clinique - PHRC - Angers 1996), the AFD (Research Grant 2004) and Association Diabète Risque Vasculaire (ADRV, Paris, France).

The ISIS cohort (Nantes, Paris St Louis and Toulouse) was supported by a joint grant 'ALLIANCE' from Inserm (the French Institute of Health and Medical Research) and the French affiliate of the NovoNordisk Foundation, and by the French Ministry of Health (PHRC).

Duality of interest SH has served as a consultant and/or on advisory panels for Astra Zeneca and Bristol-Myers Squibb and has received honoraria or speaking fees from Astra Zeneca, Bristol-Myers Squibb, Abbott, Boehringer Ingelheim, Eli Lilly, Janssen, Merck Sharpe \& Dohme, Novartis, Novo Nordisk, Sanofi, Servier and Takeda, has received research grants from Abbott and Takeda and has received travel grants from Janssen, Astra Zeneca, Bristol-Myers Squibb, Merck Sharpe \& Dohme and Sanofi. BC has served as a consultant and/or on advisory panels for Amgen, AstraZeneca, DebioPharm, Janssen, Eli Lilly, Genfit, Novo Nordisk, Sanofi, Regeneron and Takeda and has received research funding from Sanofi. MM has served as a consultant and/or on advisory panels for Abbott, Merck Sharpe \& Dohme, Novo Nordisk, Sanofi and Servier, has received honoraria or speaking fees from Abbott, Eli Lilly, Merck Sharpe \& Dohme, Novo Nordisk, Sanofi, Servier and Takeda and has received research grants from Abbott, Merck Sharpe \& Dohme, Novartis, Novo Nordisk, Sanofi and Servier. RR has served as a consultant and/or on advisory panels for Astra Zeneca, Bristol-Myers Squibb, Novo Nordisk, Sanofi, Merck, Janssen and Abbvie, has received honoraria or speaking fees from Astra Zeneca, Bristol-Myers Squibb, Abbvie,
Boehringer Ingelheim, Janssen, Merck, Novartis, Novo Nordisk and Sanofi, has received research grants from Sanofi and Janssen and has received travel grants from Janssen, Astra Zeneca, Bristol-Myers Squib and Sanofi. GC has served as a consultant and/or on advisory panels for Boehringer Ingelheim, Janssen, Eli Lilly, Medronic, Novo Nordisk and Sanofi, has received honoraria or speaking fees from Eli Lilly, Novo and Sanofi and has received research grants from Novartis and Sanofi. PJS has received travel grants from Servier, Novo Nordisk and Roche Diagnostics. JFG has received consulting honoraria from AstraZeneca, Bristol-Myers Squibb, Eli Lilly, Novartis, Novo Nordisk, SanofiAventis and Servier. JFG has received speaking fees from Astra Zeneca, Bristol-Myers Squibb, Eli Lilly, Novartis, Novo Nordisk and Servier and has received research grants from Eli Lilly, Novo Nordisk and Sanofi.

All other authors declare that there is no duality of interest associated with their contribution to this manuscript.

The analysis and interpretation of the data has been carried out without the participation of any of these organisations and companies.

Contribution statement $\mathrm{SH}, \mathrm{BC}, \mathrm{GC}, \mathrm{A}-\mathrm{AK}, \mathrm{JFG}, \mathrm{PG}, \mathrm{P}-\mathrm{JS}$ and MM researched data. $\mathrm{SH}$ and $\mathrm{EG}$ performed analysis. RR, KM and $\mathrm{EF}$ contributed to the analysis of data. FF contributed to the analysis and interpretation of data. SH wrote the manuscript and contributed to the discussion; all other authors critically revised the manuscript. All authors approved the final version to be published. SH is the guarantor of this work.

\section{References}

1. The expert Committee on the diagnosis and classification of diabetes mellitus (1997) Report of the expert committee on the diagnosis and classification of diabetes mellitus. Diabetes Care 20:1183-1197

2. Groop PH, Thomas MC, Moran JL et al (2009) The presence and severity of chronic kidney disease predicts all-cause mortality in type 1 diabetes. Diabetes 58:1651-1658

3. Orchard TJ, Secrest AM, Miller RG, Costacou T (2010) In the absence of renal disease, 20 year mortality risk in type 1 diabetes is comparable to that of the general population: a report from the Pittsburgh Epidemiology of Diabetes Complications Study. Diabetologia 53:2312-2319

4. Afkarian M, Sachs MC, Kestenbaum B et al (2013) Kidney disease and increased mortality risk in type 2 diabetes. J Am Soc Nephrol 24:302-308

5. Ninomiya T, Perkovic V, de Galan BE et al (2009) Albuminuria and kidney function independently predict cardiovascular and renal outcomes in diabetes. J Am Soc Nephrol 20:1813-1821

6. Gale EA (2006) Declassifying diabetes. Diabetologia 49: 1989-1995

7. Hasslacher C, Ritz E, Wahl P, Michael C (1989) Similar risks of nephropathy in patients with type I or type II diabetes mellitus. Nephrol Dial Transplant 4:859-863

8. Levey AS, Inker LA, Matsushita K et al (2014) GFR decline as an end point for clinical trials in CKD: a scientific workshop sponsored by the National Kidney Foundation and the US Food and Drug Administration. Am J Kidney Dis 64:821-835

9. Skupien J, Warram JH, Smiles AM, et al (2012) The early decline in renal function in patients with type 1 diabetes and proteinuria predicts the risk of end-stage renal disease. Kidney Int 82:589-597

10. Hadjadj S, Péan F, Gallois Y et al (2004) Different patterns of insulin resistance in relatives of type 1 diabetic patients with retinopathy or nephropathy: the Genesis France-Belgium Study. Diabetes Care 27:2661-2668

11. Marre M, Jeunemaitre X, Gallois Y et al (1997) Contribution of genetic polymorphism in the renin-angiotensin system to the development of renal complications in insulin-dependent diabetes: 
Genetique de la Nephropathie Diabetique (GENEDIAB) study group. J Clin Invest 99:1585-1595

12. de Hauteclocque A, Ragot S, Slaoui Y et al (2014) The influence of sex on renal function decline in people with type 2 diabetes. Diabet Med 31:1121-1128

13. Lièvre M, Marre M, Chatellier G et al (2000) The non-insulindependent diabetes, hypertension, microalbuminuria or proteinuria, cardiovascular events, and Ramipril (DIABHYCAR) Study: design, organization, and patient recruitment. Control Clin Trials 21:383-396

14. Marre M, Lievre M, Chatellier G, Mann JF, Passa P, Menard J (2004) Effects of low dose ramipril on cardiovascular and renal outcomes in patients with type 2 diabetes and raised excretion of urinary albumin: randomised, double blind, placebo controlled trial (the DIABHYCAR study). BMJ 328:495

15. Levin A, Stevens PE (2014) Summary of KDIGO 2012 CKD Guideline: behind the scenes, need for guidance, and a framework for moving forward. Kidney Int 85:49-61

16. Levey AS, Stevens LA, Schmid CH et al (2009) A new equation to estimate glomerular filtration rate. Ann Intern Med 150:604-612

17. Adler AI, Stevens RJ, Manley SE, Bilous RW, Cull CA, Holman RR (2003) Development and progression of nephropathy in type 2 diabetes: the United Kingdom Prospective Diabetes Study (UKPDS 64). Kidney Int 63:225-232

18. Forsblom C, Harjutsalo V, Thorn LM et al (2011) Competing-risk analysis of ESRD and death among patients with type 1 diabetes and macroalbuminuria. J Am Soc Nephrol 22:537-544

19. Biesenbach G, Hubmann R, Grafinger P, Stuby U, Eichbauer-Sturm G, Janko O (2000) 5-year overall survival rates of uremic type 1 and type 2 diabetic patients in comparison with age-matched nondiabetic patients with end-stage renal disease from a single dialysis center from 1991 to 1997. Diabetes Care 23:1860-1862

20. Pugh JA, Medina R, Ramirez M (1993) Comparison of the course to end-stage renal disease of type 1 (insulin-dependent) and type 2 (non-insulin-dependent) diabetic nephropathy. Diabetologia 36:1094-1098

21. Eriksson J, Forsen B, Haggblom M, Teppo AM, Groop L (1992) Clinical and metabolic characteristics of type 1 and type 2 diabetes: an epidemiological study from the Narpes community in western Finland. Diabet Med 9:654-660

22. Penfornis A, Personeni E, Tiv M et al (2012) Quality of care of patients with type 1 diabetes: population-based results in a French region. Diabetes Metab 38:436-443

23. Saulnier PJ, Gand E, Ragot S et al (2014) Association of serum concentration of TNFR1 with all-cause mortality in patients with type 2 diabetes and chronic kidney disease: follow-up of the SURDIAGENE Cohort. Diabetes Care 37:1425-1431

24. Pezzolesi MG, Poznik GD, Mychaleckyj JC et al (2009) Genomewide association scan for diabetic nephropathy susceptibility genes in type 1 diabetes. Diabetes 58:1403-1410

25. Maeda S, Araki S, Babazono T et al (2010) Replication study for the association between four Loci identified by a genome-wide association study on European American subjects with type 1 diabetes and susceptibility to diabetic nephropathy in Japanese subjects with type 2 diabetes. Diabetes 59:2075-2079

26. Hur J, Nair V, Brosius F, Kretzler M, Feldman EL (2015) Shared transcriptional networks between diabetic peripheral neuropathy and nephropathy in murine models of diabetes [abstract]. Diabetes 64(suppl 1):A50 